\title{
Quantum Transport in Open Nanostructures
}

\author{
I. V. ZOZOULENKO* and K.-F. BERGGREN \\ Department of Physics and Measurement Technology, Linköping University, S-58183 Linköping, Sweden
}

\begin{abstract}
Electron transport was studied in an open square quantum dot with a dimension typical for current experiments. A numerical analysis of the probability density distribution inside the dot was performed which enabled us to unambiguously map the resonant states which dominate the conductance of the structure. It was shown that, despite of the presence of dot openings, transport through the dot is effectively mediated by just a few (or even a single) eigenstates of the corresponding closed structure. In a single-mode regime in the leads, the broadening of the resonant levels is typically smaller than the mean energy level spacing, $\Delta$. On the contrary, in the many-mode regime this broadening typically exceeds $\Delta$ and has an irregular, essentially non-Lorentzian, character. It was demonstrated that in the latter case eigenlevel spacing statistics of the corresponding closed system are not relevant to the averaged transport properties of the dot. This conclusion seems to have a number of experimental as well as numerical verifications.
\end{abstract}

Keywords: Quantum dots, resonant states, conductance fluctuations

In nanoscaled semiconductor quantum dots, electron motion is confined in all spatial dimensions and the lateral shape of the dot can be controlled by an applied gate voltage [1-6]. In high quality samples at low temperatures electron transport is ballistic, i.e., large-angle elastic scattering events occur only at the boundaries of the structure and the phase coherence length well exceeds the dimension of the device. During recent years a great deal of effort has been focused on the transport properties of ballistic microstructures. In particular, both the statistical properties of the conductance fluctuations $[1-4,7-10]$ as well as geometry-specific, non-averaged features of the magnetoresistance of quantum dots $[1,4,5,9,11]$ have been extensively studied.

Transport properties of open microstructures are often analyzed on the basis of the known characteristics of the spectrum of the corresponding isolated system. However, when the dot becomes open, eigenlevels interact and acquire a finite broadening due to the possibility for electrons to escape from the dot via lead openings. With several propagating modes in the leads, this broadening might well exceed the mean energy level separation, $\Delta$, resulting in an overlap of a vast number of resonances. Also, the presence of dot openings may cause a significant distortion of

\footnotetext{
* Corresponding author.
} 
corresponding eigenstates. Under these circumstances it is not a priori evident whether a discussion of transport through an open dot on the basis of the properties of the Hamiltonian of the closed structure is still meaningful. To the best of our knowledge, up to now no direct theoretical calculations on the actual broadening of the resonant levels for the open dots in the transmissive regime are available.

Besides, in the current literature there exists a number of conflicting reports on the effects of leads on the character of electron dynamics in open systems (chaotic vs regular). In particular, [12] shows that the statistics of the spectra for open dots are exactly the same as those of the corresponding closed system. At the same time, results $[9,10,13]$ suggest that the leads attached to the dot may change the level statistics, so that transition to chaos can occur in a nominally regular system. On the contrary, Wang et al. [8] conclude that the openness of the dot makes chaotic scattering non-essential.

In this paper, on the basis of our direct mapping of resonant states performed for an open square dot, we hope to contribute to the clarification of some fundamental issues in this context.

The system under investigation is a relatively large square dot with the side $L=1 \mu \mathrm{m}$ which is typical for current experiments. It is connected to reservoirs by quantum point contact (QPC)-like openings (leads), see Figures 1-3. For the sake of simplicity, hard wall confinement and a flat potential profile inside the dot are assumed which seems to be a good approximation for large dots [11]. We disregard effects of the soft impurity potential due to remote donors as well as inelastic scattering events.

Conductance through the dot in perpendicular magnetic field $B$ at finite temperature is calculated within the Landauer-Büttiker formalism [14], where a transmission probabilities and wave functions were computed by making use of the recursive Green function technique [15]. Analyzing the probability density distribution inside the dot we are in a position to identify resonant energy

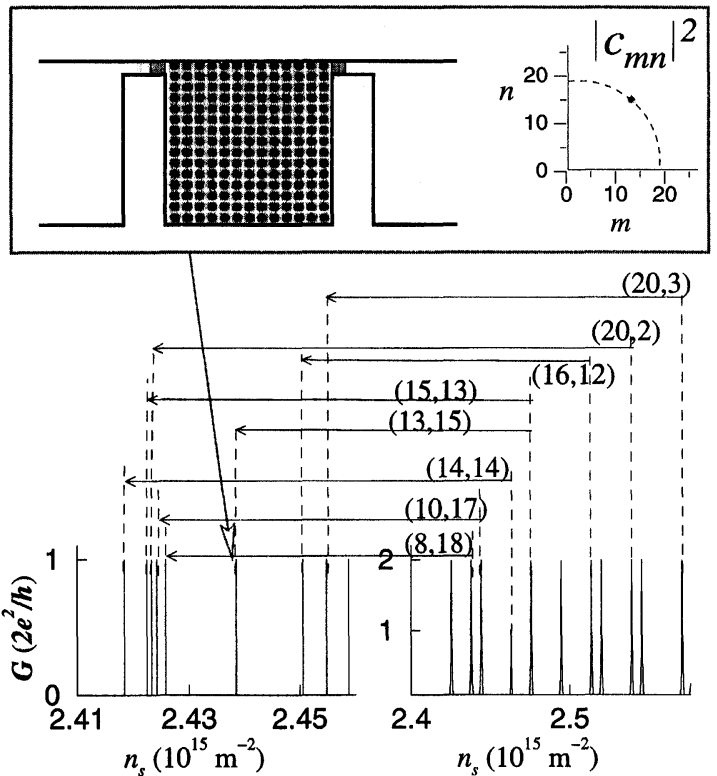

FIGURE 1 Lower left: The conductance of the square dot (schematically depicted in the upper panel) in the tunneling regime as a function of the sheet electron density $E_{F} m^{*} / \pi \hbar^{2}$. Temperature $T=0$. Shadow regions in the leads represent tunneling barriers with the height exceeding the Fermi energy. Lower right: Eigenenergy levels of the isolated dot. Height of the peaks represents the degree of degeneracy (1 or 2). Upper panel shows the calculated probability density distribution $\left|\Psi_{m n}\right|^{2}$ inside the dot for one of the tunneling peaks (left) and the corresponding numerical results for the coefficients $\left|c_{m n}\right|^{2}$ calculated on the basis of Eq. (1) (right). Dashed lines indicate the circle with the radius $r=k_{F} L / \pi$. A similar analysis has been done for the rest of the peaks and the correspondence between eigenstates of the isolated square and resonant levels of the dot is indicated by the arrows. Quantum numbers of the resonant states, $(m, n)$, are shown in the parenthesis. In the case under consideration the side of the dot was chosen to be $L=0.5 \mu \mathrm{m}$.

states which effectively mediate transport through the dot at a given $E_{F}$. To do this, we numerically expand the solution of the scattering problem in the open dot, $\Psi(x, y ; E)$, in the set of eigenstates of the closed dot, $\psi_{m n}=\frac{2}{L} \sin \frac{\pi m x}{L} \sin \frac{\pi n y}{L}$ (with eigenenergies $\left.\varepsilon_{m n}=\hbar^{2} / 2 m^{*}\left(k_{m}^{2}+k_{n}^{2}\right) ; k_{m}=\frac{\pi m}{L}, k_{n}=\frac{\pi n}{L}\right)$

$$
\Psi(x, y ; E)=\frac{2}{L} \sum_{m} \sum_{n} c_{m n}(E) \sin \frac{\pi m x}{L} \sin \frac{\pi n y}{L} .
$$

Coefficients $c_{m n}$ represent the contributions of the eigenstates $m, n$ in the total wave function. 


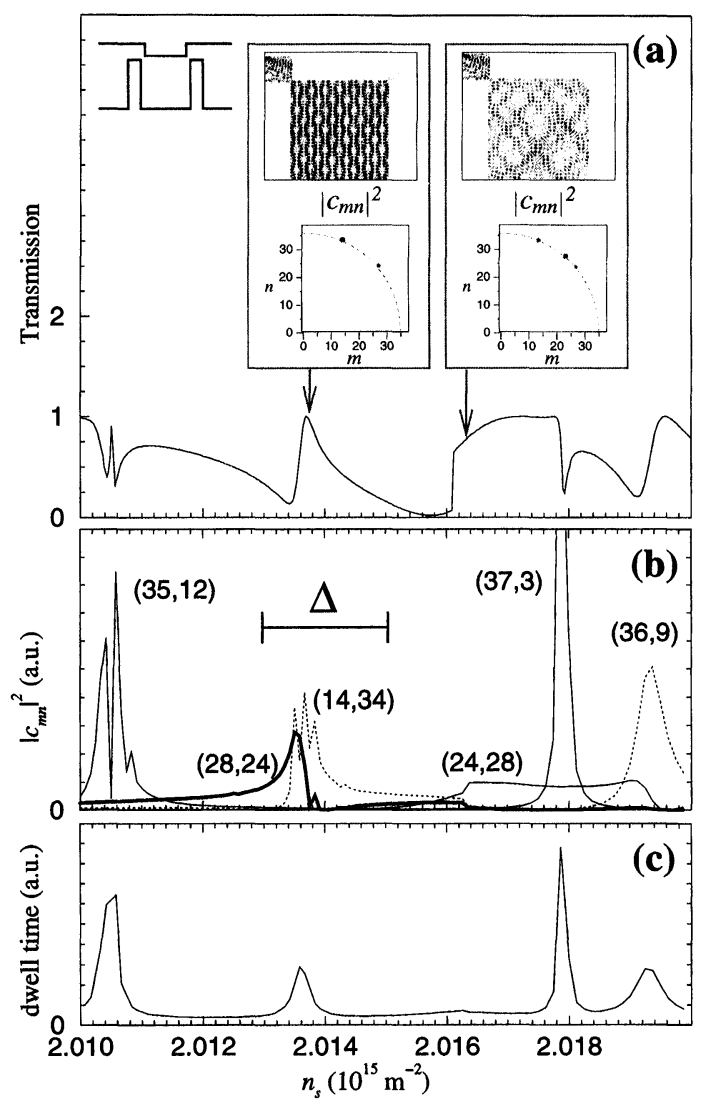

FIGURE 2 The conductance of the square dot (schematically depicted in the inset) as a function of the sheet electron density $n_{s}=E_{F} m^{*} / \pi \hbar^{2}$. The side of the $\operatorname{dot} L=1 \mu \mathrm{m}$; temperature $T=0$. The lead openings support one propagating mode. Insets show calculated $\left|\Psi_{m n}\right|^{2}$ inside the dot for two representative values of $n_{s}$ and the corresponding numerical results for the coefficients $\left|c_{m n}\right|^{2}$ calculated on the basis of Eq. (1). Dashed lines indicate the circle with the radius $r=k_{F} L / \pi$. (b) Dependence of the coefficients $\left|c_{m n}\right|^{2}$ identifying the dominant resonant states with the quantum numbers $(m, n)$ on the sheet electron density $n_{s}$. A horizontal bar indicates the mean energy level spacing $\Delta$. (c) Dwell time of the dot.

In what follows we focus on the three different transport regimes, namely the tunneling, the single-mode, and the many-mode regimes.

Tunneling regime Figure 1(a) shows conductance of the square dot in a regime when the dot is weakly coupled to the leads. Each conductance peak corresponds to an excitation of one single resonant energy level which effectively mediates transport at the given Fermi energy. Near its maximum, each peak is characterized by the

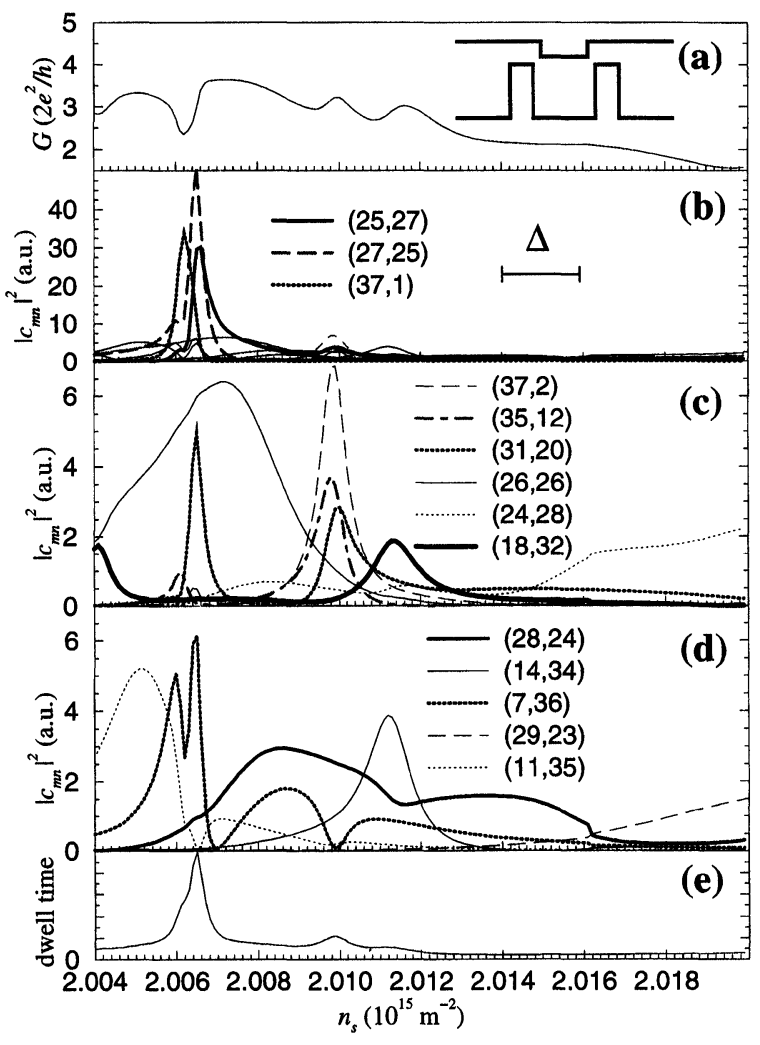

FIGURE 3 The conductance of the square dot (see inset) in a many-mode regime in the lead openings, $N=5$. The side of the $\operatorname{dot} L=1 \mu \mathrm{m}$; temperature $T=0$. (b) Coefficients $\left|c_{m n}\right|^{2}$ identifying the dominant resonant states with the quantum numbers $(m, n)$. A horizontal bar indicates the mean energy level spacing $\Delta$. (c), (d) The same as (b) but in a refined scale. (The contribution from the three dominant states indicated in (b) is not shown). (e) Dwell time of the dot.

Lorentzian shape, in accordance to the BreitWigner formalism. The positions of the peaks are shifted with respect to the corresponding eigenenergy levels of the isolated square (cf. Figs. 1(a) and (b)).

Single-mode regime Figure 2(a) shows the conductance of the square dot where lead openings are adjusted to support one propagating mode. Here the pattern of the probability density distribution $\left|\Psi_{m n}\right|^{2}$ exhibits a complicated structure, where eigenstates of the isolated square are not easily recognized. Thus, a numerical analysis on the basis of Eq. (1), in contrast to the tunneling case, is essential. Calculating the expansion coefficients 
$c_{m n}$, we find that, at the given $k_{F}$, only those coefficients associated with the circle in the $k$-space with the radius $R=k_{F} \approx \sqrt{2 m^{*} \varepsilon_{m n}} / \hbar=\pi \sqrt{m^{2}+n^{2}}$ $/ L$ are distinct from zero, see Figure 2(a). (Note that non-vanishing contributions from other coefficients would indicate that eigenstates of the isolated dot are essentially distorted by the lead openings such that a discussion of the transport in open structure in terms of eigenstates of the isolated dot does not make sense). Typically, we find that only a few (and sometimes even a single) coefficients give a dominant contribution. A broadening of the resonant levels due to the effect of the dot openings in the $k$-space is less than the distance between neighboring eigenstates whose quantum numbers differ by one, $\Delta k=\left|k_{n}-k_{n \pm 1}\right|$ $=\left|k_{m}-k_{m \pm 1}\right|=\pi / L$. Therefore, we conclude that despite the presence of dot openings, transport through the structure is still effectively mediated by a few eigenstates of the corresponding closed dot with the eigenenergies lying in close proximity to the Fermi energy, $\varepsilon_{m n}=E_{F}$.

Calculating the coefficients $c_{m n}$ as a function of the Fermi energy, we extract information about the lead-induced broadening of the energy levels of the dot. A contribution of the dominant resonant energy states is shown in Figure 2(b). The mean energy level spacing, $\Delta=2 \pi \hbar^{2} / m^{*} L^{2} \sim 70 \mathrm{mK}$, is indicated by a horizontal bar. In contrast to the tunneling regime, the lineshape of $\left|c_{m, n}(E)\right|^{2}$ can be non-Lorentzian. Moreover, different states are characterized by different broadenings and they may overlap with each other. However, a broadening (half-width) of the resonant energy levels is typically less than $\Delta$. Therefore, transport measurements at very low temperatures in a single-mode regime in the leads may probe a single resonant energy level of the dot.

A comparison between Figures 2(a) and (b) shows that features in the conductance of the dot are related to excitations of the particular eigenstates of the square. However, this correspondence is rather complicated: different eigenstates can be responsible for opposite features in the dot conductance (dips and peaks).
Many-mode regime As the lead openings become wider, a number of the resonant states excited in the dot increases. Nevertheless, like in the singlemode regime, at the given Fermi energy, a non vanishing contribution comes only from the coeffcients which lie in the closest proximity to the circle with the radius $k_{F}$ in the $k$-space. Therefore, even in a many-mode regime, transport through an open structure is still effectively mediated by eigenstates of the corresponding closed dot. A broadening of the resonant energy levels increases with an increase of the lead openings. In Figure 3 wave function patterns are analyzed on the basis of Eq. (1) and contributions from dominant states are shown in a representative interval of the Fermi energy. Typically, several states dominate transport at a given $E_{F}$. A broadening of the energy levels has, as a rule, a complicated essentially nonLorentzian character with half-width being different for different states. In contrast to the single mode regime, a half-width of the resonant energy levels is typically larger than the mean energy level spacing, $\Delta$. Comparing the conductance of the dot, Figure 3; and the dependence $c_{m n}=c_{m n}\left(E_{F}\right)$, one can trace a certain correspondence between the two. However, because many eigenstates typically contribute to the conductance at a given $E_{F}$, a detailed explanation of the features of the dot conductance is not possible.

In what follows we critically examine several approaches $[9,12]$ to the analysis of the statistics of the spectra of open dots. A statistical analysis of the distribution of the energies at resonances of conduction fluctuations in chaotic stadium and regular circular billiards has been performed by Ishio [9]. In both billiards the statistics follows Wigner-type distribution which was taken as an indication of the transition to chaos. (The Wignertype statistics of the nearest energy level spacing distribution is characteristic of the classically chaotic closed billiards). With regard to this analysis, the question immediately arises "does the resonance energy statistics of the conductance fluctuations reproduce the corresponding statistics of the isolated system?" We have shown above 
that even in a single mode regime, resonant energies in the conductance fluctuations only occasionally correspond to resonant eigenstates of the isolated dot. Instead, in most cases resonant energies are related to those energies when more than one state is simultaneously excited in the dot such that their mutual interference leads to the resonance behavior of the transmission coefficient. Therefore, in our opinion, Wigner-type statistics of the spacing of the conductance fluctuation resonances cannot be taken as an indication of the transition to chaos in a nominally regular but open system.

Wang et al. [12] analyzed statistics of the open system on the basis of the calculated electron dwell time, $\tau \sim \int d s\left|\Psi_{m n}\right|^{2}$, which identifies the time an electron spends inside the dot; in the above definition an integration is performed within the dot area. Statistics of the spectra were found to be exactly the same as that of the corresponding closed system. Again, a similar question arises "does the dwell time maxima (which is integrated characteristic) unambiguously identify individual resonant states of the open dot?".

Figures 2(c) and 3(c) show the calculated dwell time in the square dot in the single- and manymode regimes respectively. In a single-mode regime all maxima in the dwell time do correspond to the resonant eigenstates, (cf. Figs. 2(b) and (c)). However, dwell time does not identify resonant states unambiguously because some eigenstates are overlooked by this analysis. Nevertheless, since the number of missing states is usually small our analysis, as far as a single mode regime is concerned, tends to support the conclusion [12] that the statistics of the dwell time spectra for open dots are the same as that of the corresponding closed systems.

In a many-mode regime, our analysis strongly suggest that the resonant level spacing statistics become ill-defined. This is not only because the broadening of resonant states typically exceeds mean level spacing (for Lorentzian broadening one can still define statistics of the spacings between peak maxima). This is due to the fact that the broadening itself in many cases is essentially nonLorentzian, see Figure 3(c). For most of the resonant states the concept of statistics of the spectra does not make any sense, since it is not possible to introduce any reasonable definition of the spacing between resonances. Therefore, we conclude that for the quantum dots strongly coupled to the leads with several modes available in the lead openings, eigenlevel spacing statistics of the corresponding closed system are not relevant to the averaged transport properties of the structure.

This conclusion seems to have a number of experimental as well as numerical verifications. The difference between statistical properties of the conductance oscillations in a chaotic (stadium) and a regular (circular) dots has been studied by Marcus et al. [1]. Corresponding averaged autocorrelation functions are almost identical over the two decades of decay, although they exhibit quantitative distinctions in the tail. The data, from the similar studies of Berry et al. [2] for chaotic (circular with a bar) and regular (circular) dots, does not show any significant discrepancy over the four orders of magnitude in power. Numerical studies of autocorrelations functions for a chaotic (stadium) and a regular (circular) dots [8] in a manymode regime show the similar behavior, which is in accordance with our arguments.

To conclude, despite of the presence of dot openings, transport through the open dot is effectively mediated by just a few eigenstates of the corresponding closed structure. In a singlemode regime in the leads the broadening of the resonant levels is typically smaller than the mean energy level spacing, $\Delta$. On the contrary, in the many-mode regime the broadening exceeds $\Delta$ and has essentially a non-Lorentzian character.

\section{Acknowledgement}

I.V.Z. acknowledges a grant from the Royal Swedish Academy of Sciences. 


\section{References}

[1] Marcus, C. M., Rimberg, A. J., Westervelt, R. M., Hopkins, P. F. and Gossard, A. C., "Conductance Fluctuations and Chaotic Scattering in Ballistic Microstructures", Physical Review Letters, July 1992.

[2] Berry, M. J., Katine, J. A., Westervelt, R. M. and Gossard, A. C., "Influence of Shape on Electron Transport in Ballistic Quantum Dots", Physical Review B, December 1994.

[3] Chang, A. M., Baranger, H. U., Pfeiffer, L. N. and West, K. W., Weak Localization in Chaotic versus Nonchaotic Cavities: A Striking Difference in the Line Shape", Physical Review Letters, October 1994.

[4] Persson, M., Pettersson, J., von Sydow, B., Lindelof, P. E., Kristensen, A. and Berggren, K.-F., "Conductance Oscillations Related to the Eigenenergy Spectrum of a Quantum Dot in Weak Magnetic Field", Physical Review $B$, September 1995.

[5] Bird, J. P., Ferry, D. K., Akis, R., Ochiai, Y., Ishibashi, K., Aoyagi, Y. and Sugano, T., "Periodic Conductance Oscillations and Stable Orbits in Mesoscopic Semiconductor Billiards", Europhysics Letters, September 1996.

[6] Zozoulenko, I. V., Schuster, R., Berggren, K.-F. and Ensslin, K., "Ballistic Electrons in an Open Square Geometry: Selective Probing of Resonant-Energy Levels", Physical Review B, April 1997.

[7] Jalabert, R. A., Baranger, H. U. and Stone, A. D., "Conductance Fluctuations in the Ballistic Regime: A Probe of Quantum Chaos?", Physical Review Letters, November 1990.

[8] Wang, Y., Wang, J., Gou, H. and Roland, C., "Tunneling Through Quantum-Dot Systems: A Study of the Magneto-Conductance Fluctuations", J. Physics: Condensed Matter, August 1994.

[9] Ishio, H., "Quantum Transport and integrability in Open Billiards", J. Statistical Phyics, January 1996.

[10] Albeverio, S., Haake, F., Kurasov, P., Kuś, M. and Šeba, P., "S-matrix, resonances and wave functions for transport through billiard with leads", J. Math. Phys., October 1996.

[11] Reiman, S. M., Persson, M., Lindelof, P. E. and Brack, M., "Shell Structure of a Circular Quantum Dot in a Weak Magnetic Field", Z. Phys. B, October 1996.

[12] Wang, Y., Zhu, N., Wang, J. and Guo, H., "Resonance States of Open Quantum Dots", Physical Review B, June 1996.
[13] Berggren, K.-F. and Ji, Z.-L., "Quantum Chaos in Nanosized Billiards in Layered Two-Dimensional Semiconductor Structures", CHAOS, November 1996.

[14] Datta, S., Electronic Transport in Mesoscopic Systems (Cambridge University Press, Cambridge, 1995).

[15] Zozoulenko, I. V., Maaø, F. A. and Hauge, E. H., "Coherent Magnetotransport in Confined Arrays of Antidots”, Physical Review B, March 1996.

\section{Authors' Biographies}

Igor Zozoulenko is a senior scientist at the Department of Physics at Linköping University. His current research deals with electronic aspects of low-dimensional semiconductor structures like quantum dots, wells and related systems. During his career he has awarded fellowships from Royal Norwegian Council for Scientific and Industrial Research and Royal Swedish Academy of Sciences, and has held positions at Institute for Theoretical Physics, Kiev.

Karl-Fredrik Berggren is Professor of Theoretical Physics at the Department of Physics at Linköping University and head of the Laboratory of Theoretical Physics. In general terms his research is focused on condensed matter physics. $\mathrm{He}$ is a member of the Swedish Council for High Performance Computing, a member of the Computational Physics Board of the European Physical Society, and a member of the Condensed Matter Theory Committee at the Nordic Institute of Theoretical Physics (NORDITA), Copenhagen. 

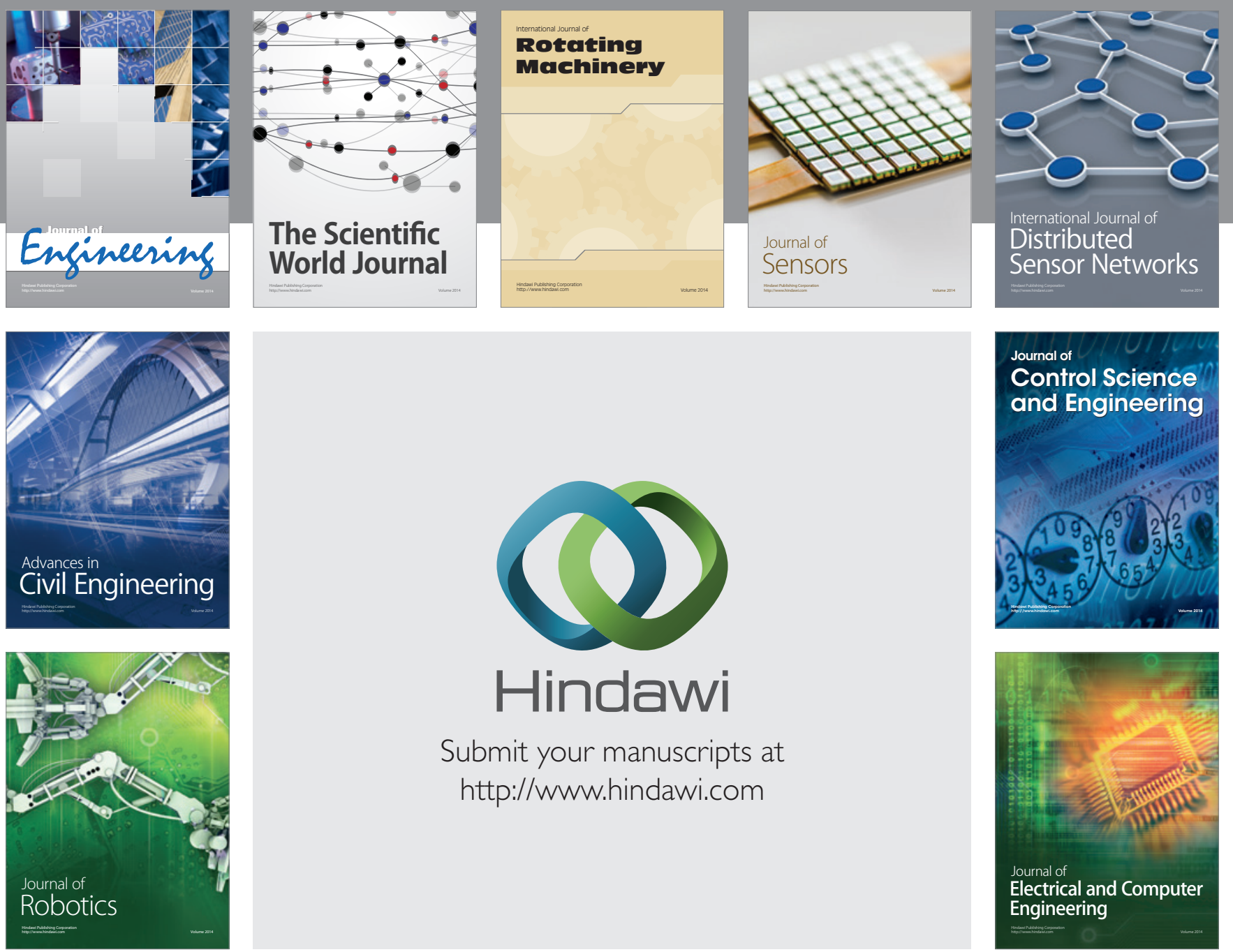

Submit your manuscripts at

http://www.hindawi.com
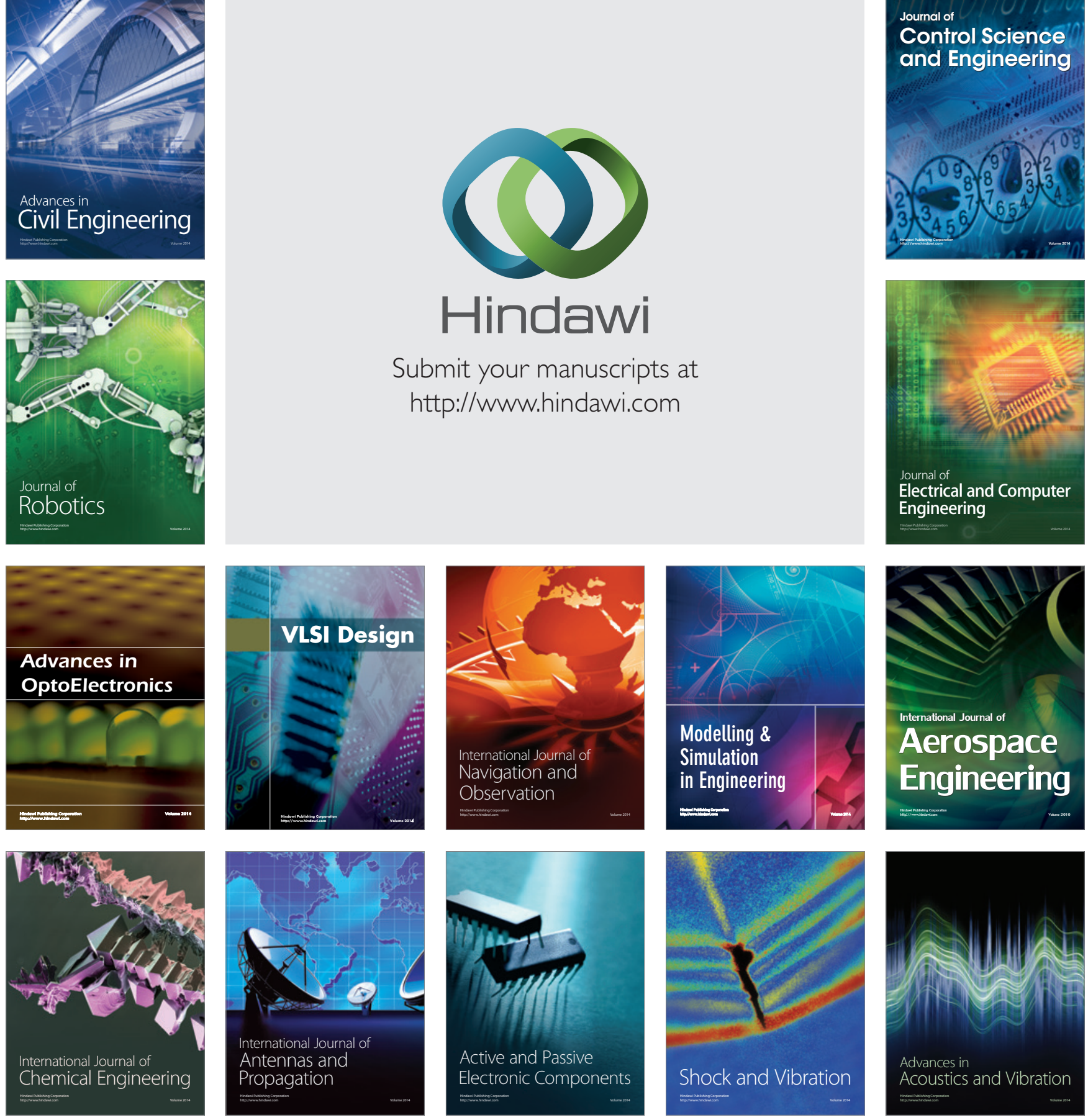